COLORECTAL CANCER

\title{
Impaired CD95 expression predisposes for recurrence in curatively resected colon carcinoma: clinical evidence for immunoselection and CD95L mediated control of minimal residual disease
}

\author{
J Sträter, U Hinz, C Hasel, U Bhanot, G Mechtersheimer, T Lehnert, P Möller
}

Gut 2005;54:661-665. doi: 10.1136/gut.2004.052696

See end of article for authors' affiliations

\section{Correspondence to:} Dr J Sträter, Department of Pathology, University of Ulm, Albert-Einstein-Allee 11,89081 Ulm, Germany; joern.straeter@ medizin.uni-ulm.de

Revised version received 27 October 2004 Accepted for publication 9 November 2004

\begin{abstract}
Background: Loss of CD95 expression in tumour cells occurs frequently in colon carcinoma and may be associated with disease progression. On the other hand, neo-expression of CD95L in tumour cells may contribute to immune evasion.

Aims: We aimed at further exploring the functional role and prognostic significance of the CD95/CD95L death inducing system in colon carcinomas.

Patients and methods: CD95 and CD95L expression was examined by immunohistochemistry in 128 RO resected UICC (International Union against Cancer) stage II/III colon carcinomas and correlated with disease free survival.

Results: CD95 expression in tumour cells was observed in only 30 carcinomas (23.4\%) whereas the others had at least a minor subpopulation of CD95 negative cells. Loss of CD95 in tumour cells was related to adverse prognosis in uni- and multivariate analysis $(p=0.046$ and $p=0.036$, respectively). Tumour infiltrating lymphocytes (TLL) were the major source of CD95L in colon carcinomas. CD95L+TIL were present in $83 \%$ of cases whereas CD95L was found in tumour cells in only $12 \%$ of cases. Moreover, a high rate of CD95L+TIL correlated with prolonged disease free survival in patients with UICC stage II $(p=0.05)$ but not in those with stage III.

Conclusions: Loss of CD95 in tumour cells may be an independent prognostic factor in colon carcinomas. The CD95L counterattack is not a relevant feature in colon carcinoma but CD95L+TIL may contribute to tumour control in the early stages of the disease, exerting a concurrent selection pressure in the direction of CD95 abrogation/resistance.
\end{abstract}

n the course of a neoplastic process, subpopulations of tumour cells evolve that behave more aggressively and contribute to progression of the disease. This may be explained, at least in part, by increased resistance of tumour cells towards apoptotic stimuli, such as irradiation and chemotherapeutic reagents. Similarly, neoplastic cells may develop strategies to evade immune defence mechanisms triggering tumour cell death. ${ }^{1}$

CD95 (Fas/APO-1), a member of the tumour necrosis factor receptor superfamily, is a death receptor able to induce apoptosis directly when oligomerised by its natural ligand, CD95L, or cross linking antibodies. ${ }^{2}{ }^{3}$ The CD95/CD95L system is a major pathway by which immune cells confer cytotoxicity $^{1}$ and may also contribute to the control of cancer. ${ }^{4}$

In the normal gut, CD95 is constitutively expressed on the basolateral surface of all epithelial cells. ${ }^{6}$ While CD95 expression is still retained in colorectal adenomas, CD95 is lost in a high percentage of colorectal carcinomas. ${ }^{7}$ Although data from animal models suggest that loss of or resistance to CD95 in colon cancer cells is associated with tumour progression, ${ }^{8-12}$ studies correlating CD95 expression with clinical outcome of patients suffering from large bowel cancer are lacking. It is also conceivable that tumour infiltrating lymphocytes (TIL) expressing CD95L may influence local tumour growth and/or metastasis. On the other hand, it has been suggested that CD95 resistant tumour cells may neo-express CD95L themselves and thus start a "counterattack" on (CD95 sensitive) TIL. ${ }^{13}$ Whether the
CD95L counterattack is a relevant feature in colon carcinoma has been a matter of debate. ${ }^{14}$

In an attempt to better understand the functional role and prognostic significance of this death inducing system in colon carcinomas, we determined the expression and tissue distribution of CD95 and CD95L in colon cancers and correlated expression patterns with disease free survival after potentially curative resection of International Union against Cancer (UICC) stage II and III colon carcinomas.

\section{PATIENTS AND METHODS \\ Patients}

The study included 128 patients with curatively resected (R0) primary colon adenocarcinomas treated between 1990 and 1996 at the Department of Surgery, University of Heidelberg, Germany. Patients with rectal carcinoma were excluded. Representative samples of tumour tissue had been snap frozen in liquid nitrogen and stored at $-80^{\circ} \mathrm{C}$. Clinical and pathological data were documented prospectively and entered into a specific tumour registry at the time of surgery and at each follow up (table 1). The registry was maintained and statistical analysis of the data was performed by a biostatistician (UH). All patients were followed according to a standard protocol which included colonoscopy, abdominal ultrasound, chest radiography, and tumour marker studies performed in the outpatient clinic or by the family physician.

Abbreviations: CD95L, CD95 ligand; TIL, tumour infiltrating lymphocytes; UICC, International Union against Cancer 
Table 1 Clinical and pathological characteristics of the colon carcinoma cases

\begin{tabular}{|c|c|c|}
\hline Characteristic & $\mathbf{n}$ & $\%$ \\
\hline Median age (y) (IQR) & 128 & $64(59-70)$ \\
\hline \multicolumn{3}{|l|}{ Sex } \\
\hline Male & 83 & 64.8 \\
\hline Female & 45 & 35.2 \\
\hline \multicolumn{3}{|l|}{ Treatment } \\
\hline Surgery alone & 104 & 81.2 \\
\hline Surgery+chemotherapy & 24 & 18.8 \\
\hline \multicolumn{3}{|l|}{ Tumour localisation } \\
\hline Caecum & 20 & 15.6 \\
\hline Ascending colon & 21 & 16.4 \\
\hline Hepatic flexure & 10 & 7.8 \\
\hline Transverse colon & 9 & 7 \\
\hline Splenic flexure & 13 & 10.2 \\
\hline Descending colon & 3 & 2.4 \\
\hline Sigmoid & 52 & 40.6 \\
\hline \multicolumn{3}{|l|}{ Tumour histology } \\
\hline Adenocarcinoma & 88 & 69 \\
\hline Mucinous carcinoma & 40 & 31 \\
\hline \multicolumn{3}{|l|}{ Grade of differentiation } \\
\hline Low grade & 98 & 76.6 \\
\hline High grade & 30 & 23.4 \\
\hline \multicolumn{3}{|l|}{ UICC stage } \\
\hline Stage II & 68 & 53.1 \\
\hline Stage III & 60 & 46.9 \\
\hline \multicolumn{3}{|l|}{ pT category } \\
\hline pT2 & 4 & 3.1 \\
\hline pT3 & 101 & 78.9 \\
\hline pT4 & 23 & 18 \\
\hline
\end{tabular}

$I Q R$, interquartile range; UICC, International Union against Cancer.

For this study, patients were given pseudonyms to comply with the German law for correct usage of archival tissue for clinical research. ${ }^{15}$ The study was approved by the ethics committee of the University of Ulm.

Median follow up time of patients alive at the last follow up was 96 months (interquartile range $75-113$ months). Twenty one UICC stage III patients and three UICC stage II patients received adjuvant chemotherapy outside of clinical studies according to patient or physician preference.

The five year overall survival rate of all 128 patients was $72 \%$, with 44 deaths observed in the follow up period. The five year disease free survival rate was $68 \%$, with 47 events observed during follow up. Forty one patients (32\%) developed distant metastases. Local tumour recurrence was diagnosed in six patients $(5 \%)$.

\section{Immunohistochemistry}

For immunohistochemistry, $4 \mu \mathrm{m}$ serial frozen sections containing representative tumour tissue were fixed in acetone for 10 minutes. Sections were incubated for $60 \mathrm{~min}-$ utes with the following monoclonal antibodies diluted in phosphate buffered saline at appropriate concentrations: the mouse antihuman CD95 antibody, clone APO-1 (Dako, Copenhagen, Denmark) and the mouse antihuman CD95L antibody, clone G247-4 (BD Biosciences Pharmingen, San

Table 2 Expression of CD95 and CD95L in tumour cells and tumour infiltrating lymphocytes (TIL)

\begin{tabular}{|c|c|c|c|}
\hline & \multirow{2}{*}{$\frac{\text { CD95 (n= 128) }}{\text { Tumour }}$} & \multicolumn{2}{|c|}{ CD95L $(n=127)$} \\
\hline & & Tumour & TIL \\
\hline Score 0 & $13(10.2 \%)$ & 112 (88.2\%) & 21 (16.5\%) \\
\hline Score 1 & $33(25.8 \%)$ & $13(10.2 \%)$ & 46 (36.2\%) \\
\hline Score 2 & $23(18.0 \%)$ & $1(0.8 \%)$ & 50 (39.4\%) \\
\hline Score 3 & $29(22.7 \%)$ & $1(0.8 \%)$ & $10(7.9 \%)$ \\
\hline Score 4 & $30(23.4 \%)$ & 0 & 0 \\
\hline
\end{tabular}

Diego, California, USA). The sensitivity and specificity of the CD95L antibody used in this study was assessed recently. ${ }^{16}$ Binding sites of primary antibodies were visualised using the Dako EnVision kit (Dako) according to the manufacturer's instructions. Finally, sections were faintly counterstained with Harris' haematoxylin and mounted with glycerol gelatin. Negative controls were performed by omission of the primary antibody.

While staining for CD95 was carried out on all 128 cases, CD95L immunohistochemistry could only be performed on 127 specimens for technical reasons.

Stained slides were analysed by two experienced pathologists (JS, PM) who had no prior knowledge of the clinical data. Antigen expression was assessed in carcinoma cells and TIL separately. TIL were identified on morphological grounds. Based on the estimated percentage of positive cells, staining results were divided into five categories: $0=$ no positive cells; $1=$ less than $40 \%$ positive cells; $2=40-60 \%$ positive cells; $3=$ more than $60 \%$ but less than $100 \%$ positive cells, and $4=100 \%$ positive cells.

\section{Statistics}

SAS software (release 8.02; SAS Institute, Inc. Cary, North Carolina, USA) was used for statistical analyses. The distribution of age at operation and follow up time of patients alive are presented as median (interquartile range). The Mann-Whitney $U$ test was used to compare the age distribution between subgroups of patients. Comparisons between UICC stage and expression of CD95 and CD95L were performed by the two tailed Fisher's exact test or the $\chi^{2}$ test, as appropriate. Disease free survival from the date of colon resection was calculated by the Kaplan-Meier estimate. ${ }^{17}$ Patients alive without local or distant recurrence at the last follow up were censored, as were two patients who died of other causes after 101 and 118 months and one patient who was lost to follow up after three months. Expression of CD95 and CD95L was dichotomised for disease free survival analysis. The log rank test was performed to compare disease free survival time distributions. Univariate and multivariate hazard ratio estimates and corresponding 95\% confidence intervals were computed using Cox proportional hazards regression analysis. ${ }^{18}$ As the overall test, the likelihood ratio test of the final model was presented. A p value of $\leqslant 0.05$ was considered statistically significant.

\section{RESULTS}

CD95 expression in carcinoma cells is correlated with disease free survival of colon carcinoma patients

Although CD95 was shown to be lost in a subset of colon carcinomas, its influence on tumour progression and patient outcome has not been well documented. We therefore studied CD95 expression in carcinoma cells using

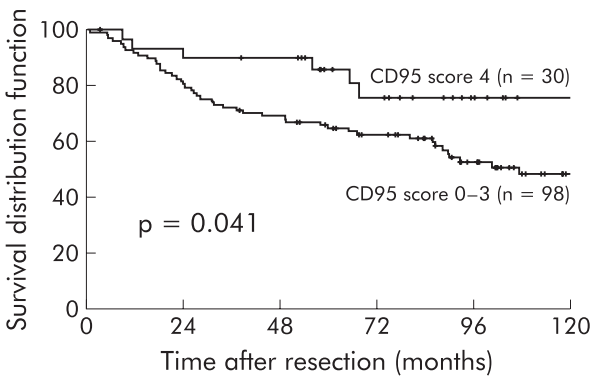

Figure 1 Kaplan-Meier estimates of disease free survival after RO resection of 128 colon carcinomas dependent on CD95 expression (score 0-3 v score 4). 
Table 3 Results of univariate and multivariate analyses of disease free survival

\begin{tabular}{|c|c|c|c|c|c|c|}
\hline \multirow[b]{2}{*}{ Variable } & \multicolumn{3}{|c|}{ Univariate analysis } & \multicolumn{3}{|c|}{ Multivariate analysis } \\
\hline & HR & $95 \% \mathrm{Cl}$ & p Value & $\mathrm{HR}$ & $95 \% \mathrm{Cl}$ & p Value \\
\hline \multicolumn{7}{|l|}{ Age } \\
\hline Continuous & 1.05 & $1.02-1.09$ & $<0.001$ & 1.07 & $1.03-1.1$ & $<0.001$ \\
\hline PT category & & & & & & \\
\hline $\begin{array}{l}\text { pT4 }<\text { pTT2-3 } \\
\text { UICC stage }\end{array}$ & 2.31 & $1.24-4.28$ & 0.008 & 3.11 & $1.65-5.86$ & $<0.001$ \\
\hline Stage III $v$ stage II & 1.30 & $0.76-2.25$ & 0.34 & 1.41 & $0.80-2.48$ & 0.23 \\
\hline \multicolumn{7}{|l|}{ Treatment } \\
\hline Surgery+chemotherapy $v$ surgery alone & 1.14 & $0.57-2.29$ & 0.70 & na & na & 0.40 \\
\hline \multicolumn{7}{|l|}{ Sex } \\
\hline Female $v$ male & 0.99 & $0.56-1.75$ & 0.96 & na & na & 0.67 \\
\hline $\begin{array}{l}\text { CD95 } \\
\text { Positive } v \text { negative }\end{array}$ & & & & & & \\
\hline Positive $v$ negative & 0.42 & $0.18-0.99$ & 0.047 & 0.40 & $0.17-0.94$ & 0.036 \\
\hline
\end{tabular}

immunohistochemistry and correlated the results with disease free survival of the patients.

The results of the immunohistochemical stainings are summarised in table 2 . Only 30 cases $(23.4 \%)$ showed CD95 expression in all tumour cells lacking a CD95 negative subpopulation (score 4). While CD95 expression in 23 $(18.0 \%)$ and $29(22.7 \%)$ cases was assigned to scores 2 and 3 , respectively, and was thus still detectable in a significant percentage of tumour cells, another 33 cases $(25.8 \%)$ expressed CD95 in only a minor subpopulation of cancer cells (score 1). Thirteen carcinomas (10.2\%) lacked CD95 completely (score 0 ).

Next, univariate and multivariate Cox proportional hazards regression analyses were performed with clinicopathological factors, including median age, sex, UICC stage, T category, adjuvant treatment, and expression of CD95 in tumour cells. When prognosis was analysed with regard to CD95 expression on tumour cells, score 4 cases were found to be associated with a significantly longer disease free survival compared with tumours with a CD95 negative subpopulation (score $0-3)$ ( $p=0.041$; fig 1$)$. This was also confirmed by multivariate analysis including age, pT category, UICC stage,

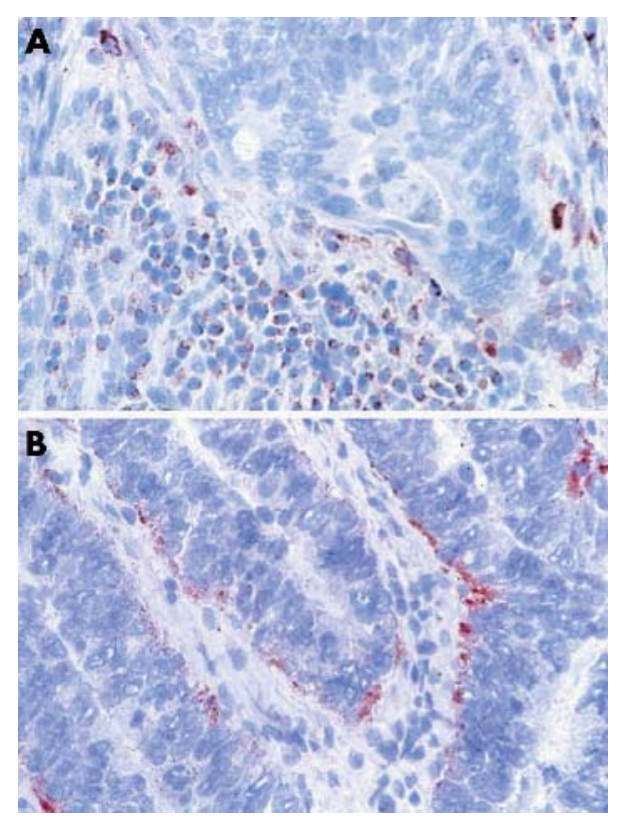

Figure 2 Immunohistochemical analysis of CD95L expression in colon cancer. (A) CD95L expression in tumour infiltrating lymphocytes. (B) Infrequent example of CD95L expression in carcinoma cells. and CD95 expression, suggesting that CD95 expression in tumour cells is an independent prognostic factor in colon carcinomas (likelihood ratio test: $\chi^{2}=31.1$, degrees of freedom 4, p<0.0001) (table 3).

\section{Tumour infiltrating lymphocytes (TIL) are the main expressors of CD95L in colon carcinomas}

Next we addressed the question of which types of cells expressed CD95L in colon carcinomas. To that end, CD95L expression was first analysed by immunohistochemistry in 127 colon carcinomas. The results are summarised in table 2 .

The bulk of CD95L+ cells were lymphocytic cells within the tumour stroma (fig 2A). Only 21 cases (16.5\%) were completely devoid of CD95L+TIL. In another 46 cases $(36.2 \%)$, CD95L+ cells made up only a minor subpopulation of TIL (score 1). However, almost half of the carcinomas (60 cases, $47.3 \%$ ) showed a considerable percentage of lymphocytes within the tumour stroma expressing CD95L (scores 2 and 3 ).

Importantly, we found CD95L only rarely expressed in the neoplastic cell population: as few as 15 of 127 cases ( $11.8 \%)$ contained CD95L+ carcinoma cells and, in the majority of these, CD95L was rather weakly expressed in a small subpopulation of tumour cells (score 1). Only two cases $(1.6 \%)$ showed a more prominent fraction of CD95L+ carcinoma cells (scores 2 and 3, respectively) (fig 2B). Moreover, the normal colonic epithelium included at the edge of the tumour in 73 cases completely lacked CD95L (not shown).

\section{High numbers of CD95L+TIL correlate with disease free survival of patients with localised but not regionally metastasised colon carcinoma}

Expression of CD95L in tumour cells and TIL was correlated with prognosis of colon carcinoma patients. Interestingly, in the univariate analysis of all 127 cases, CD95L expression in tumour cells (fig 3A) or TIL (not shown) was not associated with a statistically significant effect on disease free survival. However, the situation changed dramatically when UICC stages II and III were analysed separately. In the 68 carcinomas which were UICC stage II, a high number of CD95L+TIL was accompanied by prolonged disease free survival, with this just reaching statistical significance $(\mathrm{p}=0.05)$ (fig 3B). On the other hand, there was a trend towards an even poorer prognosis in patients with tumours rich in CD95L+TIL when tumours had already metastasised to regional lymph nodes (UICC stage III). However, possibly due to a lack of power in our study, this influence failed to reach statistical significance (fig 3C). 

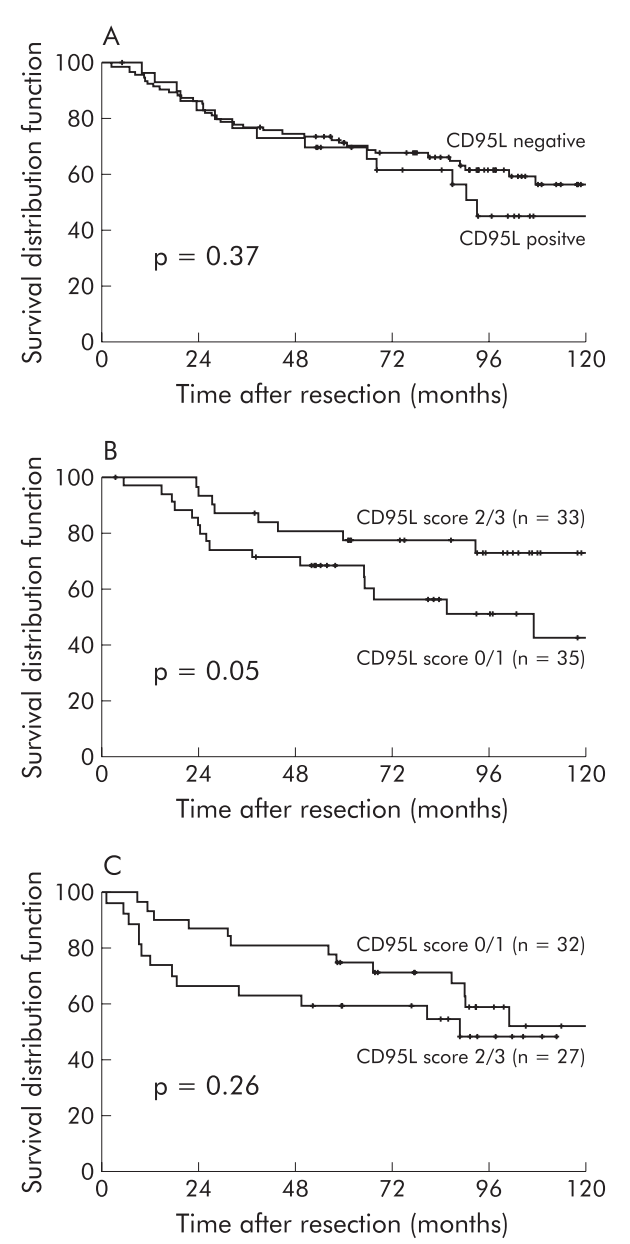

Figure 3 Kaplan-Meier estimates of disease free survival after RO resection of a colon carcinoma dependent on CD95L expression. (A) Dichotomised analysis (negative $v$ positive) according to CD95L expression in tumour cells. (B) Dichotomised analysis (score $0 / 1 v$ score 2/3) of 67 patients with carcinomas of International Union against Cancer (UICC) stage II and (C) 59 patients with carcinomas of UICC stage III dependent on CD95L expression in tumour infiltrating lymphocytes.

\section{DISCUSSION}

It was shown previously that the extent of lymphocytic infiltrate in a tumour influences the outcome of patients suffering from colorectal carcinomas, as well as other malignomas. ${ }^{19} 20$ There is now growing evidence that the CD95/CD95L system is one important pathway by which cytotoxic immune cells induce apoptosis in tumour cells and thus may contribute to tumour surveillance by TIL. ${ }^{21}{ }^{22}$ Loss of sensitivity to CD95 induced apoptosis by downregulation of CD95 expression or impaired CD95 signalling ${ }^{23}$ may therefore play an important role in immune evasion of malignant tumours.

Although its physiological role in the normal gut is not yet clear, the death receptor CD95 is constitutively expressed and functionally active in normal colon epithelial cells. ${ }^{624}$ While CD95 expression is retained in colorectal adenomas, we can confirm previous data that loss of CD95 in at least a major subpopulation of tumour cells is a feature of many carcinomas of the large bowel. ${ }^{7}$ We also demonstrate that in the majority of colon carcinomas there is a high prevalence of immune cells expressing CD95L within the tumour stroma. Taken as a whole, these observations strongly support the concept that once they have penetrated the basal membrane, neoplastic epithelial cells in the colon are subject to a CD95L driven immune attack leading to selection of CD95 negative (and resistant) subclones.

Several studies in mice indicate that resistance to CD95 induced apoptosis of tumour cells is indeed associated with increased local growth and a more aggressive phenotype. ${ }^{8-12}$ In a mouse melanoma model, disruption of the CD95 pathway was necessary and sufficient for tumour progression and metastasis. ${ }^{8}$ Although mechanisms other than reduced CD95 expression may contribute to apoptosis resistance in colon carcinoma cells (for example, loss of caspase 8 activity by inactivating mutations or expression of CFLIP), ${ }^{25} 26$ we have shown here for the first time that the emergence of CD95 negative populations of tumour cells in human colon carcinoma is associated with an adverse prognosis. This association was maintained in multivariate analysis suggesting that CD95 expression is an independent prognostic factor in colon carcinoma.

While the untransformed colon epithelium lacks CD95L in the normal and inflamed mucosa, ${ }^{24}$ we can confirm previous studies which showed that colon carcinoma cells may abnormally neo-express CD95L. ${ }^{13}$ Thus our present study is also informative with regard to the "counterattack hypothesis" which, in recent years, has been met with considerable interest. This intriguing concept is based on the idea that once they have become resistant to CD95 mediated apoptosis, tumour cells may, in turn, counterattack (CD95 sensitive) immune cells by expressing CD95L. Actually, it was shown that some CD95 resistant and CD95L expressing melanoma and colon carcinoma cell lines were able to drive cocultured $\mathrm{T}$ cells into apoptosis in vitro. ${ }^{13} 27$ Thus tumour cells may evade the immune system, not only by developing mechanisms that confer resistance to immune cell induced apoptosis, but by actively killing TIL via CD95/CD95L interactions.

Immunohistochemical data at first seemed to support the relevance of the counterattack hypothesis in vivo. O'Connell and colleagues ${ }^{28}$ found CD95L expression in all 31 cases studied. Younes and colleagues ${ }^{29}$ confirmed CD95L expression in colon carcinomas and, additionally, described increasing frequency of $\mathrm{CD} 95 \mathrm{~L}$ expression in colorectal adenomas depending on size and tubulovillous or villous morphology. However, studies showing CD95L in humans mainly relied on immunohistochemistry with CD95L antibodies of sometimes very questionable specificity. ${ }^{16}$ In our present study, we were able to detect (focally weak or moderate) CD95L expression in tumour cells in only $12 \%$ of 127 colonic adenocarcinomas, whereas it was found in TIL in the vast majority of cases $(83.6 \%)$, indicating that TIL are the main expressors of CD95L in colon carcinomas. Moreover, although our study may lack sufficient power in this respect, we did not find any correlation between CD95L expression in carcinoma cells and disease free survival. While a CD95L driven counterattack may occur in a subset of colon carcinomas, our present data suggest that, in total, its clinical significance has been overestimated.

Similar to CD95L expression in carcinoma cells, its expression in TIL did not appear to influence prognosis when UICC stages II and III were analysed together. When UICC stages were analysed separately however, it was found that patients suffering from a locally advanced carcinoma without lymph node metastases (UICC stage II) had a significantly longer disease free survival when high numbers of CD95L+TIL were present. Paradoxically, there was a trend towards an inverse correlation between the number of CD95L+TIL and survival in patients whose tumours already had metastasised to regional lymph nodes (UICC stage III) (although this correlation, possibly due to a lack of power, did not reach statistically significant levels in our study).

These data suggest a model in which a CD95L mediated immune attack on cancer cells contributes to control of 
tumour growth in earlier stages of the disease. At the same time, however, a high prevalence of CD95L+ lymphocytes within the tumour stroma may increase the selection pressure on tumour cells to develop a CD95 negative and metastatic phenotype. Evolving subclones may then be even more aggressive than metastatic clones grown in the absence of CD95L+TIL, possibly due to the fact that the latter remain under the control of CD95L mediated T cell attack at distant sites such as lymph nodes or the liver.

In the light of this model, a recent study by Liu and colleagues $^{30}$ merits attention. These authors recently showed that SW480 colon carcinoma cells cultured in the presence of the agonistic CD95 antibody, CH-11, were shown to develop a CD95 resistant phenotype and behave more aggressively in a xenograft nude mouse model. In contrast with mice however, increased aggressiveness of CD95 negative tumour cell populations in humans could not be explained by simple resistance of tumour cells towards CD95 mediated cell death, as transfection of SW480 with vFLIP was not sufficient to confer a metastatic phenotype. ${ }^{30}$ Rather, $\mathrm{CH}-\mathrm{ll}$ treated SW480 cells differed from untreated controls in terms of expression of a broad set of genes not related to CD95 signalling. ${ }^{30}$ Thus continuous exposure to CD95L may influence tumour growth and metastasis in a more complex way than simply rendering tumour cells resistant to CD95 mediated apoptosis and promoting immune evasion.

\section{ACKNOWLEDGEMENTS}

This study was supported by grants from the Deutsche Krebshilfe (Str 10-1644-St2). We thank Cornelia Herbst and Carola Dorsch for skilful technical assistance and Caroline Higginson for help with the manuscript.

\section{Authors' affiliations}

J Sträter, C Hasel, U Bhanot, P Möller, Department of Pathology,

University of Ulm, Ulm, Germany

U Hinz, T Lehnert, Section of Surgical Oncology/Department of Surgery,

University of Heidelberg, Heidelberg, Germany

G Mechtersheimer, Department of Pathology, University of Heidelberg, Heidelberg, Germany

Conflict of interest: None declared

\section{REFERENCES}

1 Itoh N, Yonehara S, Ishii A, et al. The polypeptide encoded by the cDNA for human cell surface Fas can mediate apoptosis. Cell 1991;66:233-43.

2 Oehm A, Behrmann I, Falk W, et al. Purification and molecular cloning of the APO- 1 cell surface antigen, a member of the tumor necrosis/nerve growth factor receptor superfamily. J Biol Chem 1992;267:10709-15.

3 Nagata S. Apoptosis by death factor. Cell 1997;88:355-65.

4 Peng SL, Robert ME, Hayday AC, et al. A tumor suppressor function for Fas (CD95) revealed in T cell-deficient mice. J Exp Med 1996;184:1 149-54.

5 Takeuchi T, Sasaki Y, Ueki T, et al. Modulation of growth and apoptosis response in PC-3 and LNCAP prostate-cancer cell lines by Fas. Int J Cancer 1996;67:709-14.
6 Leithäuser F, Dhein J, Mechtersheimer G, et al. Constitutive and induced expression of APO-1, a new member of the nerve growth factor/tumor necrosis factor receptor superfamily. Lab Invest 1993;69:415-29.

7 Möller P, Koretz K, Leithäuser F, et al. Expression of APO-1 (CD95), a member of the NGF/TNF receptor superfamily, in normal and neoplastic colon epithelium. Int $J$ Cancer 1994;57:371-7.

8 Owen-Schaub LB, van Golen KL, Hill LL, et al. Fas and Fas ligand interactions suppress melanoma lung metastasis. J Exp Med 1998;188:1717-23.

9 Dierbi M, Screpanti V, Catrina Al, et al. The inhibitor of death receptor signaling, FLICE-inhibitory protein defines a new class of tumor progression factors. J Exp Med 1999; 190:1025-31.

10 Medema JP, de Jong J, van Hall T, et al. Immune escape of tumors in vivo by expression of cellular FLICE-inhibitory protein. J Exp Med 1999;190:1033-8.

11 Maecker HL, Yun Z, Maecker HT, et al. Epigenetic changes in tumor Fas levels determine immune escape and response to therapy. Cancer Cell 2002;2:139-48.

12 Liu K, Abrams SI. Alterations in Fas expression are characteristic of, but not solely responsible for, enhanced metastatic competence. J Immunol 2003;170:5973-80.

13 O'Connell J, O'Sullivan GC, Collins JK, et al. The Fas counterattack: Fasmediated T cell killing by colon cancer cells expressing Fas ligand. J Exp Med 1996; 184:1075-82.

14 Favre-Felix N, Fromentin A, Hammann A, et al. The tumor counterattack hypothesis revisited: colon cancer cells do not induce $T$ cell apoptosis via the Fas (CD95, APO-1) pathway. J Immunol 2000;164:5023-7.

15 Zentrale Ethikkommission bei der Bundesärztekammer. Die (Weiter-) Verwendung von menschlichen Körpermaterialien für Zwecke medizinischer Forschung. Dtsch Ärztebl 2003;100:A1632.

16 Sträter J, Walczak H, Hasel C, et al. CD95 ligand (CD95L) immunohistochemistry: a critical study on 12 antibodies. Cell Death Differ $2001 ; 8: 273-8$.

17 Kaplan EL, Meier P. Nonparametric estimation from incomplete observations. $J$ Am Stat Assoc 1958:53:457-81.

18 Cox DR. Regression models and life tables (with discussion). J R Stat Soc B 1972;34:187-220.

19 Nacopoulou L, Azaris P, Papacharalampous N, et al. Prognostic significance of histologic host response in cancer of the large bowel. Cancer 1981;47:930-6.

20 Naito Y, Saito K, Shiiba K, et al. CD8+T cell infiltrated within cancer cell nests as a prognostic factor in human colorectal cancer. Cancer Res 1998;58:3491-4

21 Screpanti V, Wallin RP, Ljunggren HG, et al. A central role for death receptormediated apoptosis in the rejection of tumors by NK cells. J Immunol 2001;167:2068-73

22 Bergmann-Leitner ES, Abrams SI. Differential role of Fas/Fas ligand interactions in cytolysis of primary and metastatic colon carcinoma cell lines by human antigen-specific CD8+CTL. J Immunol 2000;164:4941-54.

23 von Reyher U, Sträter J, Kittstein W, et al. Colon carcinoma cells use different mechanisms to escape CD95-mediated apoptosis. Cancer Res 1998;58:526-34

24 Sträter J, Wellisch I, Riedl S, et al. CD95 (APO-1/Fas)-mediated apoptosis in colon epithelial cells: a possible role in ulcerative colitis. Gastroenterology 1997:113:160-7.

25 Kim HS, Lee JW, Soung YH, et al. Inactivating mutations of caspase-8 gene in colorectal carcinomas. Gastroenterology 2003;125:708-15

26 Ryu BK, Lee MG, Chi SG, et al. Increased expression of cFLIP(L) in colonic adenocarcinoma. J Pathol 2001;194:15-9.

27 Hahne M, Rimoldi D, Schröter M, et al. Melanoma cell expression of Fas (APO-1/CD95) ligand: implications for tumor immune escape. Science 1996;274:1363-6.

28 O'Connell J, Bennett MW, O'Sullivan GC, et al. Fas ligand expresson in primary colon adenocarcinomas: evidence that the Fas counterattack is a prevalent mechanism of immune evasion in human colon cancer. J Pathol 1998; 186:240-6

29 Younes M, Schwartz MR, Finnie D, et al. Overexpression of Fas ligand (FasL) during malignant transformation in the large bowel and in Barrett's metaplasia of the esophagus. Hum Pathol 1999;30:1309-13

30 Liu K, McDuffie E, Abrams SI. Exposure of human primary colon carcinoma cells to anti-Fas interactions influences the emergence of pre-existing Fasresistant metastatic subpopulations. J Immunol 2003;171:4161-74. 